\title{
Simultaneous ground- and satellite-based airglow observations of geomagnetic conjugate plasma bubbles in the equatorial anomaly
}

\author{
Tadahiko Ogawa $^{1}$, Eiichi Sagawa ${ }^{2}$, Yuichi Otsuka ${ }^{1}$, Kazuo Shiokawa ${ }^{1}$, Thomas I. Immel ${ }^{3}$, \\ Stephen B. Mende ${ }^{3}$, and Phil Wilkinson ${ }^{4}$ \\ ${ }^{1}$ Solar-Terrestrial Environment Laboratory, Nagoya University, Honohara, Toyokawa, Aichi 442-8507, Japan \\ ${ }^{2}$ National Institute of Information and Communications Technology, Koganei, Tokyo 184-8795, Japan \\ ${ }^{3}$ Space Sciences Laboratory, University of California, Berkeley, CA 94720, U.S.A \\ ${ }^{4}$ IPS Radio and Space Services, Haymarket, New South Wales 1240, Australia
}

(Received December 16, 2004; Revised March 17, 2005; Accepted March 18, 2005)

\begin{abstract}
We compare, for the first time, geomagnetically-conjugate plasma bubbles observed by ground-based OI 630.0-nm all-sky imagers at Shigaraki, Japan $\left(34.8^{\circ} \mathrm{N}, 136.1^{\circ} \mathrm{E}\right.$; magnetic latitude $\left.25.4^{\circ} \mathrm{N}\right)$ and Darwin, Australia $\left(12.4^{\circ} \mathrm{S}, 131.0^{\circ} \mathrm{E}\right.$; magnetic latitude $\left.22.0^{\circ} \mathrm{S}\right)$, with global-scale plasma structures $(\sim 10,000 \mathrm{~km}$ in longitude $)$ in the equatorial anomaly simultaneously detected with an OI 135.6-nm imager on the IMAGE satellite at $\sim 7$ earth radii. As found previously, global-scale plasma structures in both hemisphere imaged by IMAGE consist of an array of geomagnetically-conjugate small- to medium-scale (a few hundreds to $1000 \mathrm{~km}$ in longitude) wavy structures that move to the east at $\sim 100 \mathrm{~m} \mathrm{~s}^{-1}$. We find the following: 1) plasma bubbles detected with the allsky imagers reach an apex altitude of $\sim 1800 \mathrm{~km}$ over the geomagnetic equator while moving to the east at $\sim 100$ $\mathrm{m} \mathrm{s}^{-1}$ with spacings of 200-250 km. 2) Bubbles observed with the all-sky imagers and IMAGE are embedded within the small- to medium-scale wavy structures, and some of them are located near the crest of an enhanced electron density region associated with the wavy structures. 3) The bubbles and wavy structures that are generated near sunset slant to the west with increasing latitude in both hemispheres, and tilts do not change with longitude (i.e., local time). The results suggest that the generation and evolution of plasma bubbles are closely related to those of the small- to medium-scale plasma structures.
\end{abstract}

Key words: Equatorial ionosphere, equatorial anomaly, plasma bubble, airglow.

\section{Introduction}

Plasma bubbles are depletions in plasma in the nighttime equatorial $F$-layer. They have been explored from the ground by means of radars, airglow imagers, transequatorial HF propagation, and other instruments (e.g., Kelley, 1989), and from satellites equipped with an in situ plasma probe (e.g., Oya et al., 1986; Watanabe and Oya, 1986). Airglow imaging from space has recently become a powerful tool for studying two-dimensional structures in the equatorial and low-latitude ionosphere (Mende et al., 2000; Christensen et al., 2003). In particular, Sagawa et al. (2003) have first used an OI 135.6-nm imager onboard the IMAGE satellite, which reaches an apogee of nearly 7 earth radii $(R e)$, to find $1000 \mathrm{~km}$-scale wavy plasma structures embedded in the northern and southern equatorial anomaly regions. Then, using a large number of IMAGE images, Immel et al. (2003, 2004) have clarified some characteristics of drift velocity of these plasma structures. Kelley et al. (2003) have reported coordinated observations of plasma bubbles from space (OI 135.6-nm imager on the TIMED satellite in a near circular orbit at $630-\mathrm{km}$ altitude) and the ground (OI 777.4$\mathrm{nm}$ imager in Hawaii). They found that bubble structures seen in both the airglow maps were consistent with those

Copy right(c) The Society of Geomagnetism and Earth, Planetary and Space Sciences (SGEPSS); The Seismological Society of Japan; The Volcanological Society of Japan; The Geodetic Society of Japan; The Japanese Society for Planetary Sciences; TERRAPUB studied previously by means of radars and numerical simulations.

Otsuka et al. (2002) have shown, for the first time, that bubble structures observed with an OI 630.0-nm all-sky imager at Sata, Japan $\left(31.0^{\circ} \mathrm{N}, 130.7^{\circ} \mathrm{E}\right)$ were almost identical to those obtained simultaneously with an all-sky imager at Darwin in Australia $\left(12.4^{\circ} \mathrm{S}, 131.0^{\circ} \mathrm{E}\right)$ that is close to a geomagnetically-conjugate point of Sata. Shiokawa et al. (2004) have analyzed all-sky imaging data from both Sata and Darwin to show that temporal and spatial evolution of bubbles also exhibit good geomagnetic conjugacy in both hemispheres. These facts strongly suggest that plasma depletion regions with a maximum apex altitude of $\sim 1700$ $\mathrm{km}$ at the geomagnetic equator elongate along geomagnetic field lines.

Until now, ground- and satellite-based observations have revealed the detailed characteristics of plasma bubbles in the equatorial $F$-layer. However, our knowledge of the relationship between bubble phenomena in a limited region (as observed by, for example, an all-sky imager) and large-scale background ionospheric structure, in which bubbles are located, is still poor. In this paper we compare, for the first time, geomagnetically-conjugate plasma bubbles simultaneously observed by two ground-based OI 630.0-nm all-sky imagers in both hemispheres with two-dimensional globalscale ( $\sim 10,000 \mathrm{~km}$ in longitude) ionospheric structures imaged by the IMAGE satellite, and show that bubbles are 


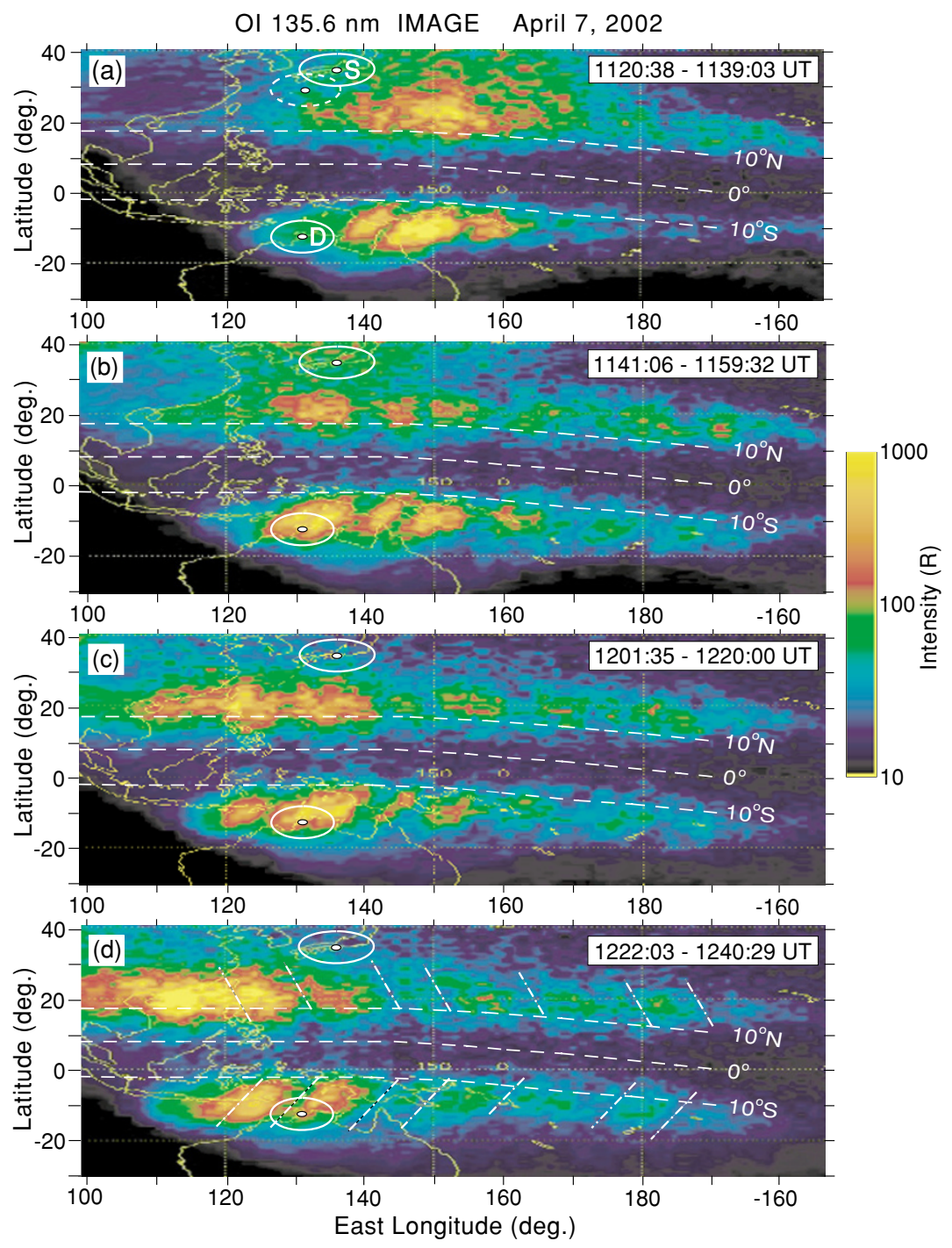

Fig. 1. OI 135.6-nm IMAGE images in geographic coordinates for four time periods on April 7, 2002. Geomagnetic latitudes are shown by the dashed curves. Dark area near the left bottom corner in each image is the region outside of the imager field of view (FOV). Positions of Shigaraki (S) and Darwin (D) are marked by the dots. Shigaraki and Darwin imager FOVs (1000 km in diameter) are also indicated. Darwin FOV mapped onto the northern hemisphere along geomagnetic fields is shown in (a) by the dashed circle. The dot-dashed lines in (d) trace slants of the airglow region.

embedded within wavy ionospheric structures with scales of few hundreds to $1000 \mathrm{~km}$.

\section{Observations and Analysis}

The IMAGE satellite has a camera for imaging the upper atmosphere at OI $135.6 \mathrm{~nm}$ (Mende et al., 2000). This camera was designed to take auroral pictures, but as demonstrated by Sagawa et al. (2003) and Immel et al. (2003, 2004), the instrument is also sensitive enough to image the low-latitude ionosphere at night. The camera on the satellite located at $7 R e$ altitude can take an image with a spatial resolution of $90 \mathrm{~km} \times 90 \mathrm{~km}$ at ionospheric altitudes and a temporal resolution of $2 \mathrm{~min}$ (satellite spin period). The exposure time for one image is $5 \mathrm{~s}$. Since, as a first approximation, the 135.6-nm volume emission rate is proportional to the square of the $F$-layer plasma density, the column emission measured by IMAGE originates mostly from a $100-\mathrm{km}$ altitude range that includes the $F$-layer peak (Sagawa et al., 2003).

All-sky CCD imagers for measuring OI 630.0-nm air- glow at $200-300 \mathrm{~km}$ altitudes in the bottomside $F$-layer have been operated at Shigaraki, Japan $\left(34.8^{\circ} \mathrm{N}, 136.1^{\circ} \mathrm{E}\right.$; magnetic latitude $\left.25.4^{\circ} \mathrm{N}\right)$ and at Darwin $\left(12.4^{\circ} \mathrm{S}, 131.0^{\circ} \mathrm{E}\right.$; magnetic latitude $22.0^{\circ} \mathrm{S}$ ). Two-dimensional airglow intensity maps with a field-of-view (FOVs) of about 1000 $\mathrm{km}$ in diameter were obtained every $5.5 \mathrm{~min}$ and $6 \mathrm{~min}$ at Shigaraki and Darwin, respectively, with an exposure time of 165 s (Shiokawa et al., 1999). The geomagneticallyconjugate point of Darwin is located at about $700 \mathrm{~km}$ southwest of Shigaraki, meaning that the Darwin imager FOV mapped onto the northern hemisphere along geomagnetic field lines partly overlaps with the Shigaraki imager FOV (see Fig. 1(a)).

\subsection{IMAGE observations}

Figure 1 shows four OI 135.6-nm images of the lowlatitude ionosphere that were obtained between 1120:381240:29 UT $(\mathrm{LT}=\mathrm{UT}+9$ hours at both Shigaraki and Darwin) on April 7, 2002, when IMAGE was located at $\sim 7$ $R e$ altitude and a magnetic latitude of $30^{\circ} \mathrm{N}$ near midnight meridian. Each image shown here combines 10 consecu- 
Ol $135.6 \mathrm{~nm}$ IMAGE April 7, 2002

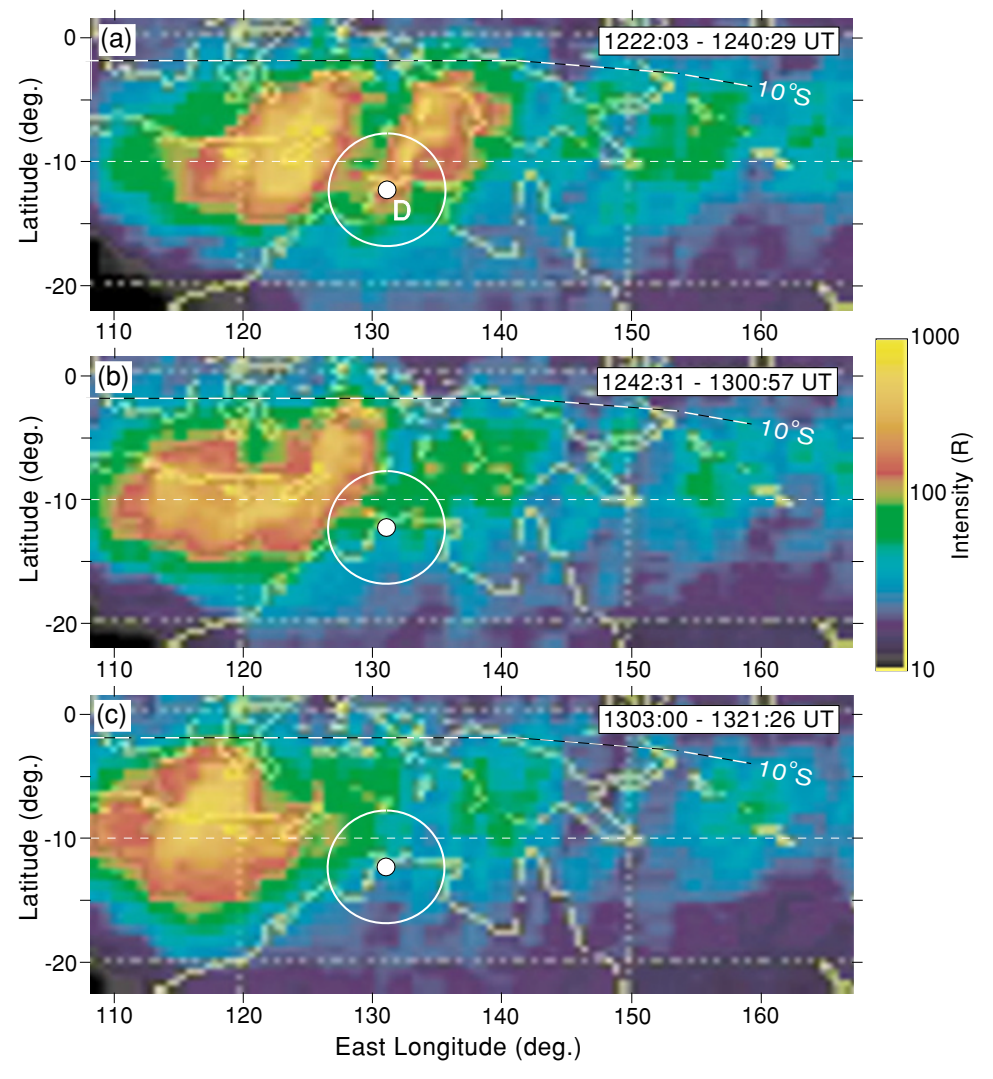

Fig. 2. Same as Fig. 1, but for three time periods. Position of Darwin (D) is marked by the white dot. Darwin imager FOV (1000 km in diameter) is also indicated by the circle.

tive images, obtained over $20 \mathrm{~min}$ (total exposure time of about $50 \mathrm{~s}$ ), to improve the signal-to-noise ratio, and is projected onto geographic coordinates by assuming an emission altitude of $350 \mathrm{~km}$. Note that because the dark area near the left bottom corner in each figure is outside of the imager's FOV, structures (if any) prior to and near sunset are unknown. The wavy airglow (electron density) structures were observed at hours between local sunset and midnight (or a little later) over a total longitudinal distance of $\sim 10,000 \mathrm{~km}$ ( $\sim 6$ hours in local time). They existed within the wide band-like airglow regions that correspond with the equatorial anomalies in both hemispheres. New bright airglow patches were successively created with time near the solar terminator, resulting in an apparent motion of the airglow patches to the west: actually the alternately bright and faint airglow regions moved to the east together, as has been demonstrated by Sagawa et al. (2003) and Immel et al. (2003, 2004).

The FOVs (1000 km in diameter) of the Shigaraki and Darwin all-sky imagers are indicated in Fig. 1. The Darwin FOV mapped onto the northern hemisphere along geomagnetic fields is shown in Fig. 1(a) by the white dashed circle. The mapped FOV partly overlaps with the Shigaraki imager FOV. A faint 135.6-nm airglow region exists within both the Darwin and Shigaraki FOVs in Fig. 1(a) (see also Fig. 7(a)). In Figs. 1(b) and 1(c), a bright airglow appears in the northern half of the Darwin FOV (see also Fig. 7(b)), whereas the airglow over Shigaraki is weak because the main equatorial anomaly is located to the south of Shigaraki. A bright airglow appears in the northeastern sky over Darwin in Fig. 1(d) (see also Fig. 2(a)). Three images taken between 1222:03 and 1321:26 UT by IMAGE are enlarged in Fig. 2 to see the detailed airglow structures in the southern hemisphere. A bright airglow region exists in the northeastern sky over Darwin in Fig. 2(a). Airglow intensities in Figs. 2(b) and 2(c), however, are weak over Darwin.

Analyzing IMAGE pictures taken on this day, Sagawa et al. (2003) have pointed out the following: (1) two airglow bands in both hemispheres show a longitudinal modulation with a scale of about $10^{\circ}(\sim 1000 \mathrm{~km})$, (2) structures in the bands are roughly in phase in both hemispheres, which suggests that the structures are geomagnetically conjugate, and (3) eastward phase velocity of the structures is $\sim 100 \mathrm{~m} \mathrm{~s}^{-1}$. In addition to these findings, we note that the bright and faint airglow regions in Figs. 1 and 2 slant to the west with increasing geomagnetic latitude in both hemispheres, and that small-scale (a few hundred $\mathrm{km}$ ) structures exist within the $1000-\mathrm{km}$ structures. As shown by the white dot-dashed lines in Fig. 1(d), the slant angles are almost constant with longitude and are very similar in the both hemispheres. We show below that plasma bubbles are collocated within the small- to medium-scale structures (a few hundreds to 1000 $\mathrm{km}$ ), and that the faint airglow regions do not always contain plasma bubbles observed by the all-sky imagers.

Using original IMAGE data with a temporal resolution of $2 \mathrm{~min}$, we generated a keogram, i.e., emission intensity at a fixed latitude plotted as a function of time and 


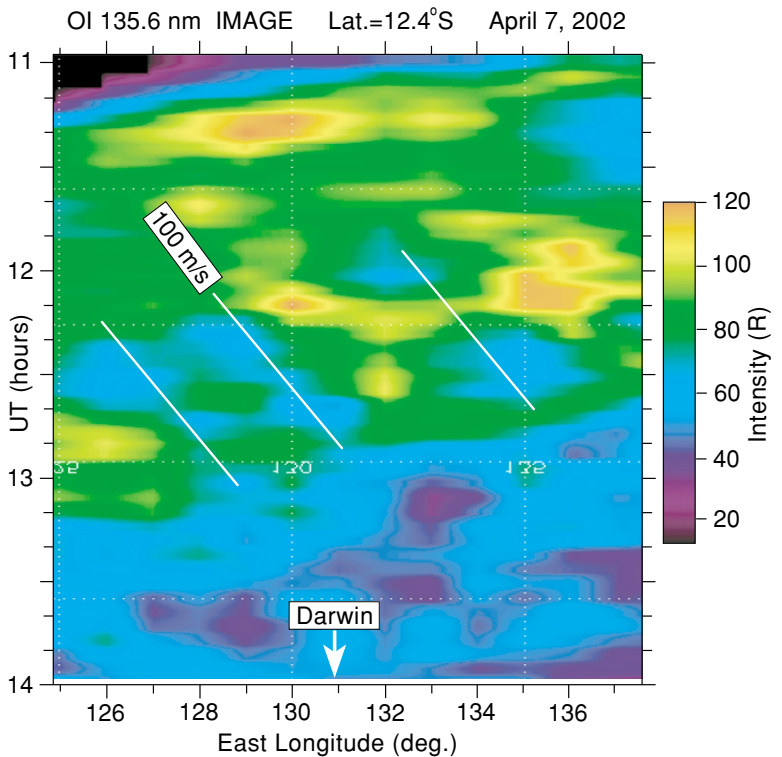

Fig. 3. Keogram of OI 135.6-nm airglow at $12.4^{\circ} \mathrm{S}$ constructed from raw IMAGE data with a temporal resolution of $2 \mathrm{~min}$.

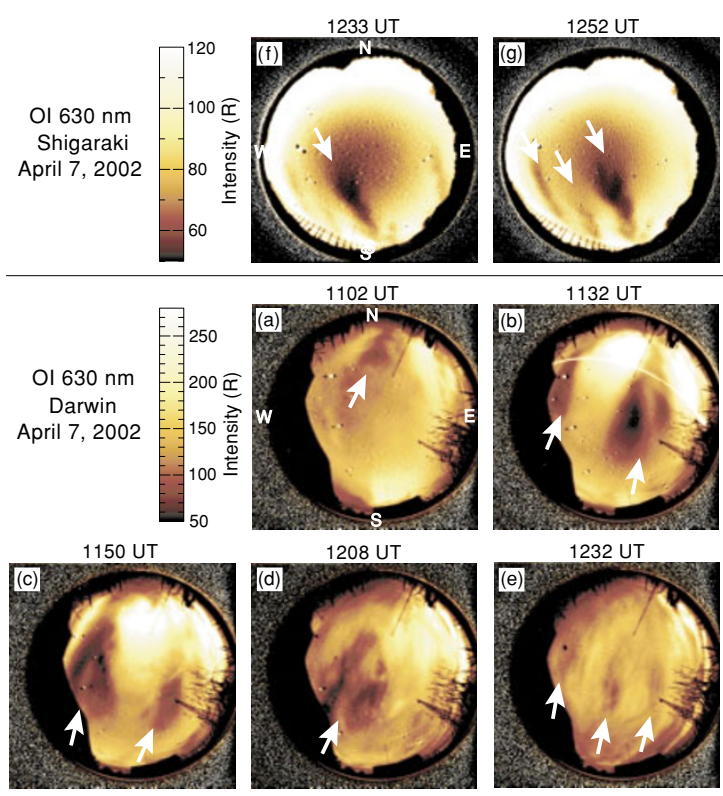

Fig. 4. Examples of OI 630-nm all-sky image at Darwin ((a)-(e)) and Shigaraki ((f) and (g)) on April 7, 2002. Plasma bubbles are pointed by the arrows. Bright area in the northeastern sky in (b), (c) and (d) is caused by clouds. The white curve in (b) is due to the passage of an airplane.

longitude. Figure 3 displays the keogram at $12.4^{\circ} \mathrm{S}$ where Darwin is located. Although it is difficult to see the motions of the airglow structures near the solar terminator at around $1100 \mathrm{UT}$ in the figure, detailed analysis shows that the motions were eastward at $220-270 \mathrm{~m} \mathrm{~s}^{-1}$ near the terminator and slowed down thereafter. After 1200 UT they moved to the east at about $100 \mathrm{~m} \mathrm{~s}^{-1}$, as shown by the white lines in the figure. Three plasma bubbles (i.e., the regions with depleted airglow intensity) with a longitudinal width of $\sim 100 \mathrm{~km}\left(\sim 1^{\circ}\right.$ in longitude) can be discerned between 1150 and $1300 \mathrm{UT}$ and between $126^{\circ}$ and $136^{\circ} \mathrm{E}(\sim 1000$ $\mathrm{km}$ in length). These bubbles are not well recognized in

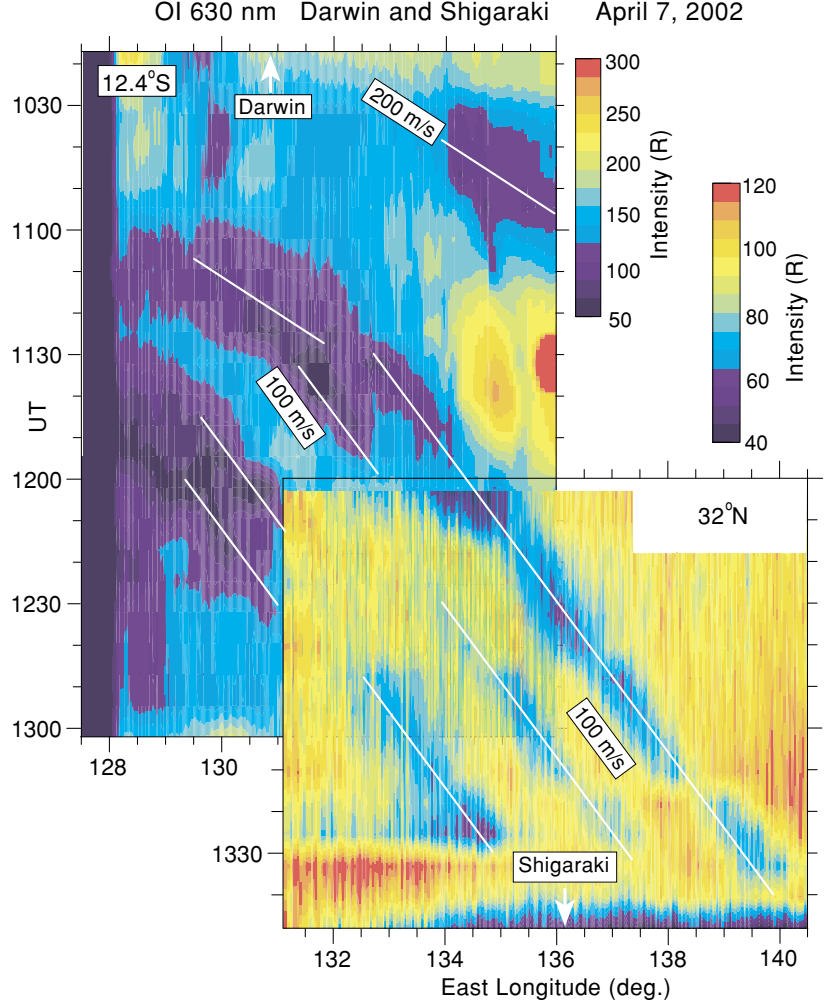

Fig. 5. Keograms of OI 630-nm airglow at $12.4^{\circ} \mathrm{S}$ and $32.0^{\circ} \mathrm{N}$ constructed from Darwin and Shigaraki all-sky images, respectively. Bright area between $134^{\circ}$ and $136^{\circ}$ east longitude and between 1110 and 1200 UT in the Darwin keogram is due to clouds.

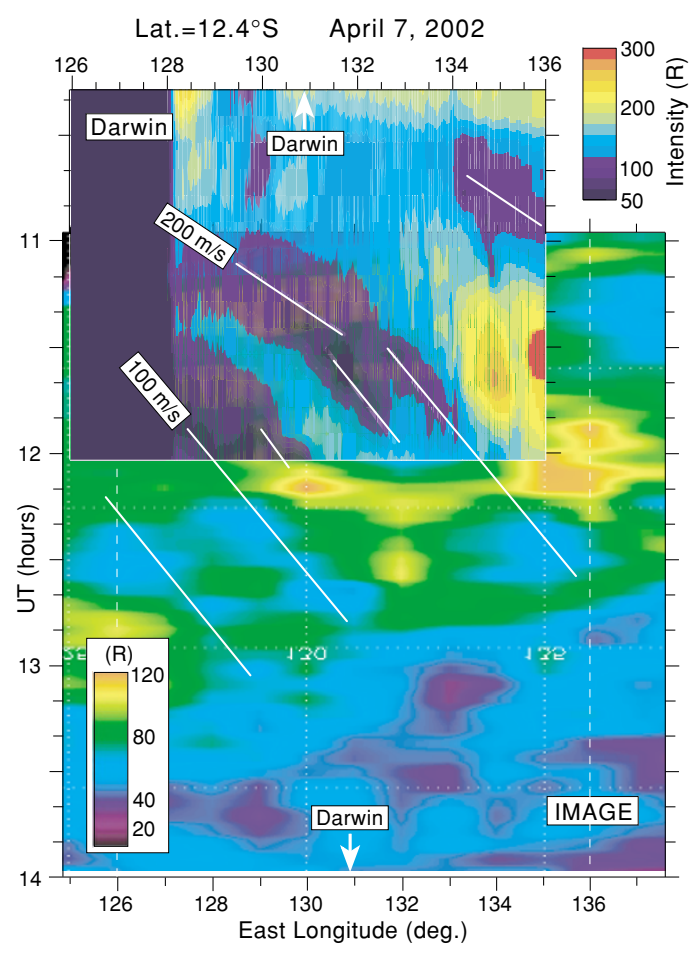

Fig. 6. Keograms of OI 630- and 135.6-nm airglow at $12.4^{\circ} \mathrm{S}$ constructed from Darwin all-sky and IMAGE images, respectively. The abscissa of the Darwin keogram is shifted by $1^{\circ}$ from that of the IMAGE keogram.

Fig. 2(a), mainly because 10 consecutive images are combined to make Fig. 2(a). From a comparison of the IMAGE 
keogram in Fig. 3 and the IMAGE pictures in Figs. 1 and 2 , we point out that these bubbles detected by IMAGE are embedded within both the bright and faint airglow regions (i.e., within the regions with high and low electron density, respectively, near the $F$-layer peak) with a medium scalelength of about $1000 \mathrm{~km}$ (see Fig. 7 in detail).

\subsection{All-sky imager observations}

Figure 4 displays examples of OI 630-nm all-sky image at Darwin (Figs. 4(a) to 4(e)) and Shigaraki (Figs. 4(f) and $4(\mathrm{~g}))$. Note that LT $=\mathrm{UT}+9$ hours at both Shigaraki and Darwin. At Darwin a plasma bubble (pointed by the white arrow) with low airglow intensity, which extends along the meridian with a narrow longitudinal width, started to appear in the northern sky prior to 1100 UT (Fig. 4(a)), and then expanded toward the south while drifting to the east. In Fig. 4(b) (1132 UT), there are two bubbles extending from the north (equator) toward the south, and the eastern bubble exhibits a bifurcation. These bubbles are located within the weak 135.6-nm airglow region in Fig. 1(a) (see also Figs. 3 and 7(a)). One reason why the 135.6-nm airglow region does not show any bubble signatures may be that the bubbles were located at altitudes lower than the $F$-layer peak. Two bubbles in Fig. 4(b) moved further to the east, as recognized in Figs. 4(c) (1150 UT) and 4(d) (1208 UT). Consulting with Figs. 1(b) and 1(c), we find that the bubbles at 1150 and 1208 UT are embedded within the bright 135.6-nm airglow region (see also Fig. 7(b)). As shown in Fig. 3, these bubbles are also detected by IMAGE. Note that the bright area in the northeastern sky, in particular, in Figs. 4(b), 4(c), and 4(d), is caused by clouds (see Fig. 5 in detail). The bright area between two bubbles in Figs. 4(b) and 4(c) may correspond to a plasma blob (i.e., localized plasma density enhancement) observed by a 630-nm all-sky imager in Brazil (Pimenta et al., 2004) and by satellites (e.g., Watanabe and Oya, 1986). The bubbles in Fig. 4(e) (1232 UT) are faint. Figures 1(d), 2(a) and 3 indicate that the 135.6-nm airglow over Darwin was becoming weak when the bubbles were faint. After 1240 UT, no bubbles were observed at Darwin, and the IMAGE airglow intensities were also weak (see Fig. 3), which suggests that the dark 135.6-nm airglow region over Darwin in Figs. 2(b) and 2(c) contain no plasma bubbles. As evident in Figs. 4(a) to 4(e), the bubbles at Darwin slant to the west with increasing latitude (e.g., Otsuka et al., 2002; Kelley et al., 2003; Shiokawa et al., 2004), a regular feature also seen in Figs. 1 and 2.

At Shigaraki the plasma bubbles started to appear at around 1140 UT (2040 LT) and disappeared after 1340 UT. Clear bubbles extending from the south toward the zenith of Shigaraki are discernible in Figs. 4(f) (1233 UT) and 4(g) (1252 UT). The maximum apex altitude of the welldeveloped bubbles at Shigaraki is about $1800 \mathrm{~km}$ at the geomagnetic equator (Otsuka et al., 2002; Shiokawa et al., 2004). The bubbles also slant to the west with increasing latitude. The background airglow intensity is higher at Darwin than at Shigaraki, because as shown in Fig. 1, the Darwin FOV is within the southern equatorial anomaly while the Shigaraki FOV is located to the north of the northern equatorial anomaly.

\subsection{Comparison between all-sky imager and IMAGE observations}

We generated a keogram from the all-sky airglow images. The 630.0-nm emission altitude is assumed to be $250 \mathrm{~km}$ in making keograms. The keograms at $12.4^{\circ} \mathrm{S}$ (Darwin) and $32.0^{\circ} \mathrm{N}\left(2.8^{\circ}\right.$ south of Shigaraki) are shown in Fig. 5. In the Darwin keogram, a bubble appeared at 1030 UT (1930 LT) near the solar terminator at around $134^{\circ} \mathrm{E}$, and moved to the east at about $200 \mathrm{~m} \mathrm{~s}^{-1}$. The bubbles that appeared at around 1100 UT moved to the east also at about 200 $\mathrm{m} \mathrm{s}^{-1}$ at the initial phase, and thereafter at about $100 \mathrm{~m} \mathrm{~s}^{-1}$. Such a change in movement can basically be explained by a temporal change of electric fields created by neutral winds in the F-layer (e.g., Rishbeth, 1971; Martinis et al., 2003). The bubbles $\left(200 \mathrm{~m} \mathrm{~s}^{-1}\right)$ between 1100 and 1130 UT and between $129^{\circ}$ and $132^{\circ} \mathrm{E}$ cannot be discerned in the IMAGE keogram in Fig. 3, perhaps because the bubbles were located at altitudes lower than the $F$-layer peak. Note that the bright area between $134^{\circ}$ and $136^{\circ} \mathrm{E}$ and between 1110 and 1200 UT in the Darwin keogram is due to clouds (see Fig. 4).

The bubbles that moved to the east at $100 \mathrm{~m} \mathrm{~s}^{-1}$ over Darwin were also detected at Shigaraki after 1140 UT. As indicated by the white lines, conjugacy of the bubbles in both hemispheres is clearly evident. The bubbles have a longitudinal width of less than $100 \mathrm{~km}$. The separations between adjacent bubbles are $200-250 \mathrm{~km}$, which are consistent with the separations seen in Fig. 3. These values are consistent with those from previous results (Otsuka et al., 2002; Shiokawa et al., 2004) and with the velocities of drifting plasma density depletions occurring within the nighttime equatorial airglow arcs that were observed with the IMAGE satellite (Immel et al., 2003, 2004).

Figure 6 compares the IMAGE keogram (Fig. 3) with the Darwin keogram (Fig. 5). In the figure, the abscissa of the Darwin keogram is shifted by $1^{\circ}$ from that of the IMAGE keogram to achieve a better coincidence between the Darwin and IMAGE bubbles. As indicated by the oblique white lines with $100 \mathrm{~m} \mathrm{~s}^{-1}$, three dark airglow regions (plasma bubbles) before 1300 UT between $126^{\circ}$ and $136^{\circ} \mathrm{E}(\sim 1000 \mathrm{~km}$ in length) in the IMAGE keogram are well connected to the $630.0-\mathrm{nm}$ bubbles seen in the Darwin keogram. This finding means that the bubbles are embedded within the 1000-km scale electron density structures detected by IMAGE. Note that the bubble centered at 1200 UT and $132^{\circ} \mathrm{E}$ in Fig. 3 corresponds well with the bubble detected there by the Darwin all-sky imager. The reasons why the bubbles in the IMAGE keogram are a little faint may be the coarse longitudinal resolution (about $90 \mathrm{~km}$ ) and the shorter exposure time $(5 \mathrm{~s})$.

No clear counterparts corresponding to the bubbles observed at Shigaraki were identified on the IMAGE keogram at $32.0^{\circ} \mathrm{N}$ (not shown) because of very low IMAGE counting rates (Fig. 1). However, we speculate that the bubbles were embedded in the northern equatorial anomaly below $30^{\circ} \mathrm{N}\left(20^{\circ} \mathrm{N}\right.$ geomagnetic $)$.

Finally, the IMAGE and Darwin images at two time periods are compared in Fig. 7. An area of the Darwin all-sky image $(1000 \mathrm{~km} \times 1000 \mathrm{~km}$ on geographic coordinates $)$ is marked on the IMAGE picture by the rectangle: note that 


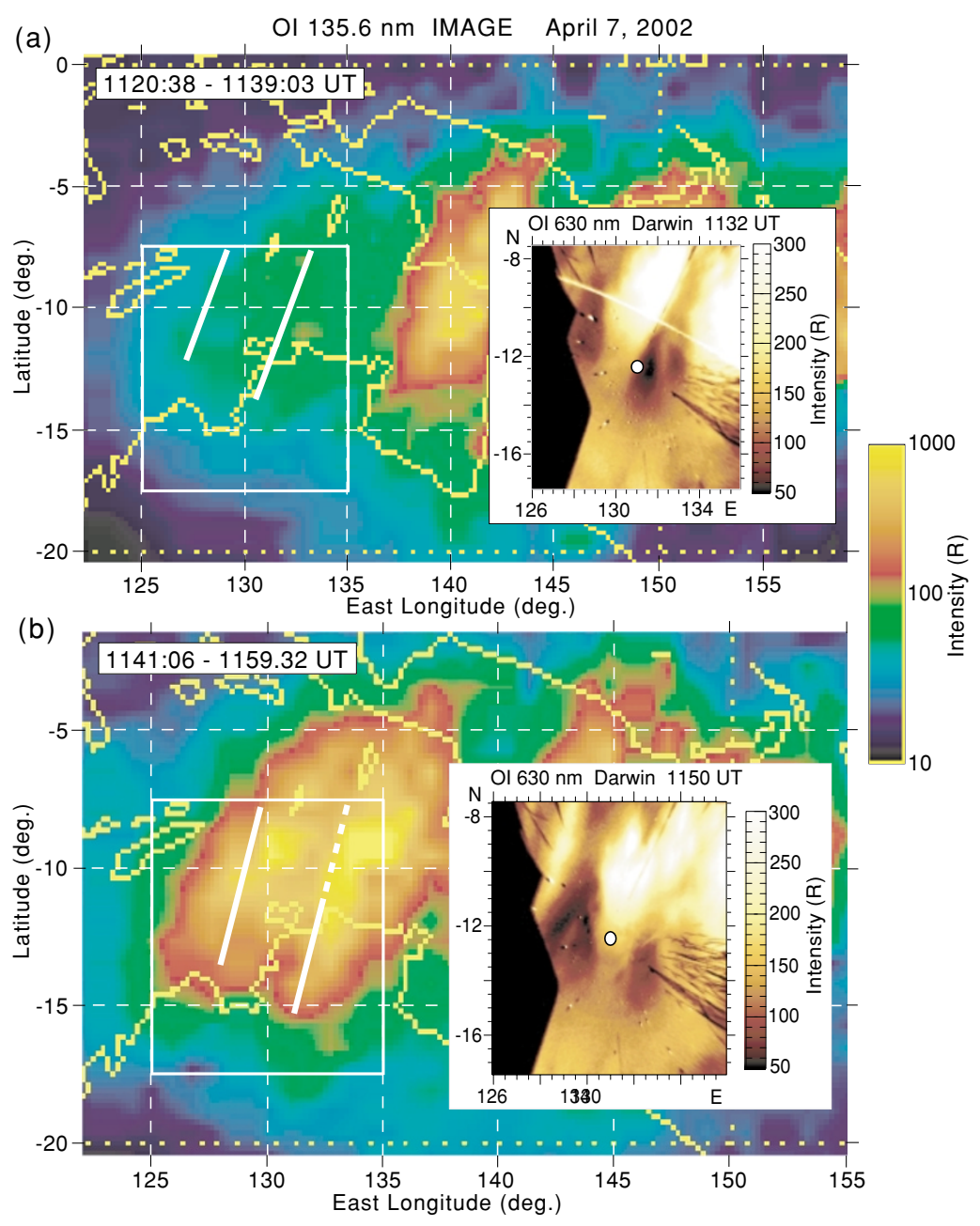

Fig. 7. OI 135.6-nm IMAGE images and OI 630-nm all-sky images at Darwin in geographic coordinates for two time periods on April 7, 2002. Area of the Darwin all-sky image $(1000 \mathrm{~km} \times 1000 \mathrm{~km}$ on geographic coordinates) is marked by the rectangle on the IMAGE picture (note that the rectangle is shifted to the left by $1^{\circ}$ ). The oblique thick lines on the IMAGE picture trace the locations of plasma bubbles seen in the Darwin image. The white curve on the Darwin image in (a) is due to the passage of an airplane.

the rectangle is shifted to the left by $1^{\circ}$ after Fig. 6 . Oblique white lines on each IMAGE picture trace the locations of the plasma bubbles seen in the Darwin image (the bubble locations in the Darwin images are marked by the white arrows in Fig. 4). As has been described before, the bubbles that were clearly observed at 1132 UT over Darwin are located within the faint 135.6-nm airglow region (Fig. 7(a)). The slant of the bubbles is very similar to that of the bright 135.6-nm airglow region centered at $8^{\circ} \mathrm{S}$ and $141^{\circ} \mathrm{E}$. In Fig. 7(b), two bubbles at 1150 UT over Darwin are embedded in the bright 135.6-nm airglow region, and their slant deviates a little from that of the 135.6-nm airglow region. In more detail, the two bubbles, one in the east and the other in the west of Darwin, seem to be aligned along the crests of two brightest 135.6-nm airglow regions: this situation is also true for the bubbles at 1208 UT in Fig. 4(d) (not shown). As stated before, the 630-nm bubbles over Darwin in Figs. 7(a) and 7(b) are sandwiched in the bright background airglow regions (with an emission intensity exceeding $250 \mathrm{R}$ ) that may correspond to plasma blobs with high electron density in the equatorial anomaly (Pimenta et al., 2004). However, the 135.6-nm airglow region, in which the $630-\mathrm{nm}$ bubbles are located, has a different emission in- tensity; weaker than $100 \mathrm{R}$ in Fig. 7(a) and stronger than $100 \mathrm{R}$ in Fig. 7(b). This fact may suggests that the electron density near the $F$-layer peak is higher in Fig. $7(\mathrm{~b})$ than in Fig. 7(a).

\section{Discussion}

The observations show that OI 630-nm bubbles in the Darwin all-sky images have counterparts in OI 135.6-nm airglow images simultaneously taken by IMAGE at $7 R e$ altitude. The bubbles are located within the equatorial anomalies that exhibit wavy electron density structures with longitudinal scale lengths of $\sim 1000 \mathrm{~km}$ and with an eastward movement at $\sim 100 \mathrm{~m} \mathrm{~s}^{-1}$. They appear within a high electron density region (i.e., bright 135.6-nm airglow region) and also within a low electron density region (i.e., faint 135.6-nm airglow region) in the anomaly. The detailed analysis for the former case (Fig. 7(b)) suggests that the bubbles are located around the crest of the bright 135.6$\mathrm{nm}$ airglow region. Such a feature may be consistent with the findings by Tsunoda (1983) who showed that plasma bubbles (radar backscatter plumes) existed in the west wall of large-scale $(\sim 400 \mathrm{~km})$ 'upwellings' that developed in the bottomside in the nighttime equatorial $F$-layer (see also 
Tsunoda and White, 1981), and by Kelley et al. (1981) who demonstrated that radar plumes were generated either when the bottomside $F$-layer was at the highest altitude or in the descending phase of the $F$-layer motion. The reason why the 630.0-nm bubbles were observed in the faint 135.6-nm airglow region (Fig. 7(a)) is not clear. We speculate that the electron density at around the $F$-layer peak, where most of the 135.6-nm emission originate, was low and also that the bubbles were located below the $F$-layer peak.

Both the plasma bubbles and 1000-km scale structure in both hemispheres tilt to the west with increasing latitude (Figs. 1, 2, 4 and 7). In particular, Fig. 7 indicates the tilt angle of the bubbles to be similar to that of the $1000-\mathrm{km}$ structure. Such a tilt was found in radar plume maps of plasma bubbles at Jicamarca (e.g., Woodman and LaHoz, 1976) and in plasma bubble images from the TIMED satellite (Kelley et al., 2003; Kil et al., 2004). The slant may result from a vertical shear in the eastward plasma drift velocity, i.e., if the velocity near the $F$-layer peak is faster than that at higher altitudes (e.g., Zalesak et al., 1982; Kelley et al., 2003). If this is the case for our $1000-\mathrm{km}$ scale structures, the tilt should increase more and more as they drift to the east with time, as demonstrated by Makela and Kelley (2003). However, as exemplified by the dot-dashed lines in Fig. 1(d), the tilts seen in Figs. 1 and 2 do not change with longitude (i.e., local time). On the other hand, Woodman and LaHoz (1976) assumed the bubble to move to the east slower than the background $\mathbf{E} \times \mathbf{B}$ drift, which would be the case if the neutral atmosphere moves eastward faster than the $\mathbf{E} \times \mathbf{B}$ drift, polarizing the bubble. At present we do not know whether the above-mentioned mechanisms for the plasma bubble tilt are also applicable to the tilts seen in the $1000-\mathrm{km}$ scale structures or not.

Using the ALTAIR radar, Tsunoda and White (1981) found that electron density contours in the bottomside $F$ layer were modulated in altitude over a $1200-\mathrm{km}$ east-west distance with an average east-west wavelength of $\sim 400$ $\mathrm{km}$. Using the Hinotori satellite at an altitude of $650 \mathrm{~km}$, Oya et al. (1986) found quasi periodic plasma bubbles, that is, an array of bubbles with characteristic distances of several hundred $\mathrm{km}$ to $1000 \mathrm{~km}$ (or more) (Watanabe and Oya, 1986). From AE-E satellite observations, Singh et al. (1997) showed that bubbles developed from wavy ion density structures with east-west wavelengths of $150-800 \mathrm{~km}$ in the bottomside of the $F$-layer. Transequatorial HF propagation experiments by Röttger (1973) also indicated wavelike ionospheric structures with such wavelengths. These observations strongly suggest that plasma bubbles are embedded within large-scale electron density structures, and are confirmed from our two-dimensional airglow observations by all-sky imagers and IMAGE. As pointed out by a number of researchers (e.g., Kelley et al., 1981; Singh et al., 1997 and references therein), we speculate that atmospheric gravity waves modulate the bottomside $F$-layer to generate an electron density structure with a scale of 1000 $\mathrm{km}$, and that plasma bubbles with small spatial scales $(\leq 100$ $\mathrm{km}$ ) develop through the Rayleigh-Taylor instability occurring within the 1000-km structure. Figures 1 and 3 suggest that the $1000-\mathrm{km}$ structure is also generated near the solar terminator.

\section{Conclusions}

We have studied geomagnetically-conjugate plasma bubbles observed with ground-based all-sky imagers and simultaneous global-scale ( $\sim 10,000 \mathrm{~km}$ in longitude) ionospheric structures imaged by the IMAGE satellite. We confirm that plasma bubbles observed at Darwin and Shigaraki reach a maximum altitude of $\sim 1800 \mathrm{~km}$ over the geomagnetic equator and have longitudinal scale lengths less than $100 \mathrm{~km}$ with spacings of 200-250 km. Eastward phase velocity of the bubbles is $\sim 200 \mathrm{~m} \mathrm{~s}^{-1}$ near sunset and $\sim 100$ $\mathrm{m} \mathrm{s}^{-1}$ at later hours. The global-scale plasma structures consist of an array of small- to medium-scale wavy structures (a few hundreds to $1000 \mathrm{~km}$ in longitude) that are also geomagnetically conjugate. New findings are as follows:

1) Bubbles observed with all-sky imagers and IMAGE are embedded within the small- to medium-scale structures. Some bubbles are surely located near the crest of an enhanced electron density region associated with the wavy structure, consistent with the results from previous radar observations. Bubbles were also observed in a low electron density region (faint 135.6-nm airglow region). In this case, we speculate that the electron density at around the $F$-layer peak was low and that the $630.0-n m$ bubbles existed below the $F$-layer peak.

2) Both the small- to medium-scale structures and bubbles that are generated near sunset show a slant to the west with increasing latitude in both hemispheres. The tilts do not change with longitude (i.e., local time).

The above findings suggest that the generation and evolution of plasma bubbles with a longitudinal scale of $100 \mathrm{~km}$ are closely related to those of plasma structures with scales of a few hundreds to $1000 \mathrm{~km}$. The bubbles are believed to be generated through the Rayleigh-Taylor instability. We, however, do not know how the longer-scale structures are produced near sunset. This paper has presented one case study. More simultaneous ground- and satellite-based observations of airglow are required to clarify the spatial and temporal relationship between plasma bubbles and longerscale ionospheric structures.

Acknowledgments. We thank R. T. Tsunoda for helpful discussion. The all-sky imager at Darwin is operated at the ionospheric station of the IPS Radio and Space Services, Australia. The IMAGE FUV instrument is supported by NASA at the University of California, Berkeley. This work was supported by Grant-inAid for Scientific Research $(11440145,13573006)$ and on Priority Area (764), and by the 21 st Century COE Program (Dynamics of the Sun-Earth-Life Interactive System, No. G-4) of the Ministry of Education, Culture, Sports, Science and Technology of Japan.

\section{References}

Christensen, A. B. et al., Initial observations with the Global Ultraviolet Imager (GUVI) in the NASA TIMED satellite mission, J. Geophys. Res., 108(A12), 1451, doi:10.1029/2003JA009918, 2003.

Immel, T. J., S. B. Mende, H. U. Frey, L. M. Peticolas, and E. Sagawa, Determination of low latitude plasma drift speeds from FUV images, Geophys. Res. Lett., 30(18), 1945, doi:10.1029/2003GL017573, 2003.

Immel, T. J., H. U. Frey, S. B. Mende, and E. Sagawa, Global observations of the zonal drift speed of equatorial ionospheric plasma bubbles, Ann. Geophysicae, 22, 3099-3107, 2004.

Kelley, M. C., The Earth's Ionosphere, Academic Press, San Diego, California, 1989.

Kelley, M. C., M. F. Larsen, C. LaHoz, and J. P. MuClure, Gravity wave initiation of equatorial spread F: A case study, J. Geophys. Res., 86, 
9087-9100, 1981.

Kelley, M. C., J. J. Makela, L. J. Paxton, F. Kamalabadi, J. M. Comberiate, and H. Kil, The first coordinated ground-based and space-based optical observations of equatorial plasma bubbles, Geophys. Res. Lett., 30(14), 1766, doi:10.1029/2003 GL017301, 2003.

Kil, H., S.-Y. Su, L. J. Paxton, B. C. Wolven, Y. Zhang, D. Morrison, and $\mathrm{H}$. C. Yeh, Coincident equatorial bubble detection by TIMED/GUVI and ROCSAT-1, Geophys. Res. Lett., 31, L03809, doi:10.1029/2003GL018696, 2004.

Makela, J. J. and M. C. Kelley, Field-aligned 777.4-nm composite airglow images of equatorial plasma depletions, Geophys. Res. Lett., 30(8), 1442, doi:10.1029/2003GL017106, 2003.

Martinis, C., J. V. Eccles, J. Baumgardner, J. Manzano, and M. Mendillo, Latitude dependence of zonal plasma drifts obtained from dual-site airglow observations, J. Geophys. Res., 108(A3), 1129, doi:10.1029/2002JA009462, 2003.

Mende, S. B., H. Heetderks, H. U. Frey, J. M. Stock, M. Lampton, S. P. Geller, R. Abiad, O. H. W. Siegmund, S. Habraken, E. Renotte, C. Jamar, P. Rochus, J.-C. Gerard, R. Sigler, and H. Lauche, Far ultraviolet imaging from the IMAGE spacecraft. 3. Spectral imaging of Lyman- $\alpha$ and OI $135.6 \mathrm{~nm}$, Space Sci. Rev., 91, 287-318, 2000.

Otsuka, Y., K. Shiokawa, T. Ogawa, and P. Wilkinson, Geomagnetic conjugate observations of equatorial airglow depletions, Geophys. Res. Lett., 29(15), 10.1029/2002GL015347, 2002.

Oya, H., T. Takahashi, and S. Watanabe, Observation of low latitude ionosphere by the impedance probe on board the Honotori satellite, J. Geomag. Geoelectr., 38, 111-123, 1986.

Pimenta, A. A., Y. Sahai, J. A. Bittencourt, M. A. Abdu, H. Takahashi, and M. J. Taylor, Plasma blobs observed by ground-based optical and radio techniques in the Brazilian tropical sector, Geophys. Res. Lett., 31, L12810, doi:10.1029/2004GL020233, 2004.

Rishbeth, H., Polarization fields produced by winds in the equatorial $F$ region, Planet. Space Sci., 19, 357-369, 1971.
Röttger, J., Wave-like structures of large-scale equatorial spread- $F$ irregularities, J. Atmos. Terr. Phys., 35, 1195-1206, 1973.

Sagawa, E., T. Maruyama, T. J. Immel, H. U. Frey, and S. B. Mende, Global view of the nighttime low-latitude ionosphere by the IMAGE/FUV 135.6 nm observations, Geophys. Res. Lett., 30(10), 1534, doi:10.1029/2003GL017140, 2003.

Shiokawa, K., Y. Katoh, M. Satoh, M. Ejiri, T. Ogawa, T. Nakamura, T. Tsuda, and R. H. Wiens, Development of optical mesosphere thermosphere imagers (OMTI), Earth Planets Space, 51, 887-896, 1999.

Shiokawa, K., Y. Otsuka, T. Ogawa, and P. Wilkinson, Time evolution of high-altitude plasma bubbles imaged at geomagnetic conjugate points, Ann. Geophysicae, 22, 3137-3143, 2004.

Singh, S., F. S. Johnson, and R. A. Power, Gravity wave seeding of equatorial plasma bubbles, J. Geophys. Res., 102, 7399-7410, 1997.

Tsunoda, R. T., On the generation and growth of equatorial backscatter plumes, 2, Structuring of the west wall of upwellings, J. Geophys. Res., 88, 4869-4874, 1983.

Tsunoda, R. T. and B. R. White, On the generation and growth of equatorial backscatter plumes, 1, Wave structure in the bottomside $F$ layer, $J$. Geophys. Res., 86, 3610-3616, 1981.

Watanabe, S. and H. Oya, Occurrence characteristics of low latitude ionosphere irregularities observed by impedance probe on board the Hinotori satellite, J. Geomag. Geoelectr., 38, 125-149, 1986.

Woodman, R. F. and C. LaHoz, Radar observations of $F$ region equatorial irregularities, J. Geophys. Res., 81, 5447-5466, 1976.

Zalesak, S. T., S. L. Ossakow, and P. K. Chaturvedi, Nonlinear equatorial spread $F$ : The effect of neutral winds and background Pedersen conductivity, J. Geophys. Res., 87, 151-166, 1982.

T. Ogawa (e-mail: ogawa@stelab.nagoya-u.ac.jp), E. Sagawa, Y. Otsuka, K. Shiokawa, T. I. Immel, S. B. Mende, and P. Wilkinson 